\title{
Epidemiological Study of Antibiotic Self-Medication in Tehran 1399: A Descriptive Study
}

\author{
Hesam Aldin Varpaei ${ }^{1 *}$, Hossein Esmaeili ${ }^{2}$, Mohammad Yavari ${ }^{1}$, Mohammad Mahdi Miremami ${ }^{1}$, \\ Amir Mahdi Farahani ${ }^{1}$, Faeze Esmaeili ${ }^{1}$, Saba Abachi ${ }^{1}$, Pariya Onsori ${ }^{1}$, Pedram Nouroozi ${ }^{1}$ and \\ Ali Kazemi ${ }^{3}$
}

${ }^{1}$ Bachelor of Nursing, Tehran University of Medical Sciences, Iran

${ }^{2}$ Young Researcher and Elite Clube, Tehran Medical Sciences, Islamic Azad University, Tehran, Iran

${ }^{3}$ Assistant Professor, Department of Pharmacology, Tehran University of Medical Sciences, Iran

*Corresponding author: Hesam Aldin Varpaei, Bachelor of Nursing, Tehran University of Medical Sciences, Iran

\section{ARTICLE INFO}

Received: 慧 September 30, 2020

Published: 幽 October 08, 2020

Citation: Hesam Aldin Varpaei, Hossein Esmaeili, Mohammad Yavari, Mohammad MahdiMiremami, Amir Mahdi Farahani, et al. Epidemiological Study of Antibiotic Self-Medication in Tehran 1399: A Descriptive Study. Biomed J Sci \& Tech Res 31(1)-2020. BJSTR. MS.ID.005040.

Keywords: Self-Medication; Antibiotics; Amoxicillin; Pharyngitis; Antimicrobial Resistance

\section{ABSTRACT}

Background: Self-medication is the use of medication to prevent, treat, or control the symptoms of illness, and often involves a combination of therapeutic resources, over the counter (OTC) medications, and antibiotics. Improper use of antibiotics and poor monitoring make antibiotic resistance a global health crisis, and the situation is exacerbated by the difficult discovery and production of novel antibiotics.

Materials and Methods: A cross-sectional descriptive study using questionnaire was conducted during the period from January 2020 to March 2020 targeting people who are living in Tehran.

Results: A total of 932 respondents to the questionnaire, about $86 \%$ of respondents in the past 6 months had self-medication with antibiotics. Previous experience of use and lack of time was the main cause of self-medication with antibiotics. The most selfadministered antibiotic was amoxicillin (28.25\%) followed by cephalexin (25.5\%). The most common disease / symptoms of antibiotic self-medication were sore throat (49.5\%), cough $(14.5 \%)$ and runny nose (11\%), respectively. Less than half of the subjects changed their antibiotic dose, the most important reason being accelerated It was a recovery. Roughly half of participants (44.5\%) stopped taking antibiotics after the symptoms disappeared.

Conclusion: This study showed a very high prevalence of antibiotic self-medication that can lead to the development of microbial resistance and failure therapy. Therefore, educational interventions targeting the general public, pharmacists, and health care students are of particular importance.

\section{Introduction}

Self-medication is the use of medication to prevent, treat, or control the symptoms of illness and often involves a combination of therapeutic resources and the use of over the counter (OTC) medications, including antibiotics [1]. According to the World Health Organization, self-medication means the selection and use of medications by individuals to treat self-diagnosed diseases or symptoms. Self-medication is a relatively widespread practice around the world. Developed and developing countries focus on self-medication as a component of health care policy [2]. It has also been suggested that self-medication can help prevent and treat diseases that do not require medical advice and provide a cheaper alternative to treating common diseases [3]. In fact, self-medication is a part of self-care, which has greatly contributed to the efficient use of the health care awareness system with all its legal aspects [4].Antibiotics are the first successful class of drugs that can cure diseases and are effective in treating many infections [5]. Proper 
use of antibiotics (correct medication, correct dosage, and course of treatment) can prevent Create an infection, treat, and prevent the infection from spreading. Antibiotic resistance is the ability of a pathogenic microbe to develop resistance to the effects of an antimicrobial (antibiotic) [6].

Antibiotic abuse and poor monitoring have turned antimicrobial resistance (AMR) into a global health crisis, and the situation is exacerbated by the difficult discovery and production of novel antibiotics [7].In recent years, various studies on antibiotic selfmedication have been conducted in different parts of the world to identify the prevalence, risks and factors associated with antibiotic abuse [8-11]. The World Health Organization announces the sale of drugs without Prescription is challenging in most countries [12], however, according to a 2017 study, it is estimated that more than $50 \%$ of antibiotics are purchased without a prescription and are used without a prescription in most parts of the world [13]". Various studies state that the incorrect use of antibiotics among individuals is due to lack of awareness about the authority use of antibiotics or insufficient information [14-16]. However, one study states that despite sufficient knowledge about the side effects of self-medication with antibiotic, self-medication with these drugs is common [17]. In this regard, most studies of education and community awareness are considered important and necessary in the prevention of self-medication with antibiotics $[14,18]$.

Although the World Health Organization has declared antibiotics as prescription-only drugs, research shows that factors related to the socio-cultural, behavioral, economic, and health systems play an effective role in self-medication with antibiotics in low-income countries [19-21].Most studies have shown that selfmedication and prescription by a non-physician may be due to the high cost of antibiotics, the high cost of medical consultation, and other economic factors [22,23]. Of course, even in rich countries where most medicines are free and doctors' consultations are free, self-medication with antibiotic is prevalent [18].Therefore, considering the importance of self-medication in human health and the consequences of self-medication with antibiotics (such as antibiotic resistance), the aim of this study was to investigate the prevalence and causes of self-medication with antibiotics in Tehran in 1399. Also, quality (including duration of treatment, Discontinuation, and dose adjustment of self-medication with antibiotics) are also considered.

\section{Materials and Methods}

The present study was a cross-sectional descriptive study that was conducted in the spring of 1399 (April to July 2020) on community members (including students, ordinary people, businesses, passers-by, and patients in Tehran). It was done with ethical considerations. After designing the questionnaire, ordinary people in the community entered the study with the condition of consent to participate in the project. All questionnaires were anonymous. Participants were assured of confidentiality. Questionnaires were filled out by researchers in each section. Data collection tool in this study was the use of a written questionnaire that information was completed using two methods of selffulfillment for literate participants and interviews for illiterate people. The questionnaire included questions in the form of two axes of personal information and self-medication habits with antibiotics and its causes. Each questionnaire was numbered, and coded information was extracted.

SPSS 23 software was used for statistical analysis. Data significance level was considered $5 \%$ (P-Value $<0.05$ ). Descriptive statistics analysis was performed, and the prevalence of selfmedication with antibiotics was calculated. The same descriptive analysis was used to calculate the frequency of causes of selfmedication with antibiotics and drug classes usage.

\section{Results}

In this study, 932 people in the community were studied, of which 764 (82\%) were self-employed and 168 (18\%) were other people (companion or parents) who answered the questionnaire. The mean age of the participants in this study was $62 \pm 39$. Out of the total population, $800(86 \%)$ had self-medication with antibiotics during the last 6 months. Self-reported diseases are listed in the (Table 1). Sore throat (pharyngitis) was the most important cause of antibiotic self-medication. Cough (with or without sputum) and runny nose were two relatively common causes of antibiotic selfmedication. Genital infections (such as vaginitis), diarrhea, and urinary incontinence are less common causes of self-medication with antibiotics.Several antibiotics have been used for selfmedication. Amoxicillin 226 cases, cephalexin 204 cases, cefixime 155 cases, azithromycin 126 cases, erythromycin 40 cases, ampicillin 30 cases were the most used antibiotics. The following were used by 19 cases: ceftriaxone (ampoule) 8 cases, cefazolin (ampoule) 6 cases, penicillin G 4 cases and clindamycin (ampoule) was 1 case.Participants were asked to change the dose of antibiotics used during self-medication.

Table 1: Demographic information of the participants.

\begin{tabular}{|c|c|}
\hline Gender & Frequency (percentage) \\
\hline Male & $410(44 \%)$ \\
\hline Female & $522(56 \%)$ \\
\hline \multicolumn{2}{|c|}{ Education } \\
\hline $\begin{array}{c}\text { High school and less } \\
\text { Less than 3 million tomans per } \\
\text { month }\end{array}$ & $438(47 \%)$ \\
\hline $\begin{array}{c}\text { More than 3 million tomans per } \\
\text { month }\end{array}$ & $494(53 \%)$ \\
\hline Without Income & $494(53 \%)$ \\
\hline
\end{tabular}




\begin{tabular}{|c|c|}
\hline \multicolumn{2}{|c|}{ Self-medication with Antibiotics } \\
\hline Yes & $376(47 \%)$ \\
\hline NO & $424(53 \%)$ \\
\hline
\end{tabular}

$(43.25 \%)$ said they did, and $354(44.25 \%)$ said they did not change their dose.100 cases (12.5\%) also stated that they did not know about this. Participants were then asked why the dose of the drug was changed. The results are shown in (Tables 2-4).The most common reason for increasing or decreasing the dose of the drug was to accelerate the recovery process. Also, reducing the side effects of the drug such as diarrhea, nausea and heartburn and insufficient medication were the second and third reasons for changing the dose of the drug.Participants were asked when to discontinue antibiotic self-medication.357 patients (44.5\%) after the disappearing of symptoms, 258 patients (32.5\%) after drug running out,100 patients $(12.5 \%)$ after completing the treatment period and 85 patients $(10.5 \%)$ discontinued antibiotics after a few days, regardless of the outcome. Respondents were then asked "after completing the treatment period" what source they learned about the treatment period. 38 people experienced previous doctor visits, 37 people with medication information (catalog or brochure), 17 people consulted a pharmacist, and 8 other family members experienced antibiotic treatment.

Table 2: Frequency distribution, by different causes of selfmedication with antibiotic.

\begin{tabular}{|c|c|c|}
\hline Causes & Frequency & Percentage \\
\hline Previous use experience & 161 & 20.125 \\
\hline Save time (lack of time) & 151 & 18,875 \\
\hline Advice from family members & 117 & 14,625 \\
\hline Other reasons & 90 & 11.25 \\
\hline High price of doctors' visits & 88 & 11 \\
\hline Crowded medical centers & 85 & 10,625 \\
\hline Lack of trust in doctors & 58 & 7.25 \\
\hline $\begin{array}{c}\text { Remote distance from doctor } \\
\text { or clinic }\end{array}$ & 30 & 3.75 \\
\hline Pharmacist recommendation & 20 & 2.5 \\
\hline Total & 800 & $100 \%$ \\
\hline
\end{tabular}

Table 3: Frequency distribution of disease which self-medicated with antibiotics.

\begin{tabular}{|c|c|}
\hline Disease/symptom & Frequency(percentage) \\
\hline Sore throat (pharyngitis) & $396(49.5 \%)$ \\
\hline Cough & $115(14.5 \%)$ \\
\hline runny nose & $82(11 \%)$ \\
\hline Fever & $62(8 \%)$ \\
\hline Tooth infection & $49(6 \%)$ \\
\hline nausea and vomiting & $34(4 \%)$ \\
\hline eye infection & $20(2.5 \%)$ \\
\hline Genital infection & $19(2 \%)$ \\
\hline Diarrhea & $13(1.5 \%)$ \\
\hline
\end{tabular}

\begin{tabular}{|c|c|}
\hline $\begin{array}{c}\text { Urinary tract infection (urinary } \\
\text { incontinence) }\end{array}$ & $10(1 \%)$ \\
\hline Total & $800(100 \%)$ \\
\hline
\end{tabular}

Table 4: Frequency distribution of antibiotic dose changing

\begin{tabular}{|c|c|}
\hline Cause of dose changing & Frequency (percentage) \\
\hline Accelerate the recovery of the disease & $197(57 \%)$ \\
\hline Reduce the side effects of the drug & $57(16.5 \%)$ \\
\hline Insufficient medication & $52(15 \%)$ \\
\hline Exacerbation of symptoms or disease & $40(11.5 \%)$ \\
\hline Total & $346(100 \%)$ \\
\hline
\end{tabular}

\section{Discussion}

The prevalence of antibiotic self-medication in different countries and regions varies from $31 \%$ to $47.6 \%$ [9,15-17]. In the present study, the prevalence of self-medication with antibiotics in the last 6 months was about $86 \%$, which is significant. It seems that the difference in the prevalence rate of self-medication may be due to differences in the demographic characteristics of the study samples, research methods, data collection tools, and the definition of antibiotic self-medication. Antibiotic self-medication is a complex phenomenon among individuals that is driven by a wide range of determinants [24].Several reasons for self-medication have been identified in various studies. The most common reason for self-medication is mild illness, followed by the cost of a doctor's visit, previous experience of use, long waiting time to see a doctor, and lack of time and health insurance [25]. In our study, the three main reasons for self-medication with antibiotics were previous experience with specific antibiotics (20.125\%) followed by lack of time (18.875\%) and family members' recommendations (14.625\%). The results of a similar study confirm these reasons [9].A study in Saudi Arabia shows that penicillins and macrolides are the most widely used antibiotics [26], relatively similar results in our study showed that penicillins, macrolides and cephalosporins were the most commonly used antibiotics.

Amoxicillin and cephalexin are the two most commonly used antibiotics in this study. Amoxicillin is the most widely used antibiotic for self-medication in many countries [9,18,22,27]. Amoxicillin and potassium amoxicillin / clavulanate are considered as effective and safe first-line antibiotics for upper respiratory tract infections such as pharyngitis and otitis media [28,29]. It seems that high use of amoxicillin may be limited due to its low cost, easy availability, and limited side effects [30]. Amoxicillin and macrolides are also the most common antibiotics prescribed and patients tend to use these prescriptions as a reference for similar diseases in the future [31,32]. Also, since the most common disease treated with antibiotics was sore throat or pharyngitis, and amoxicillin is a good treatment for pharyngitis, amoxicillin had the highest rate of selfmedication. Although many cases of sore throat or pharyngitis are related to viral infections and need not to be treated with antibiotics, this study is limited to confirming the bacterial infection 
of self-treated pharyngitis.In this study, antibiotics were commonly used for sore throats, cold symptoms such as cough, rhinorrhea, and fever.This is consistent with other studies, and several studies confirm that the most reported cases of antibiotic self-medication included sore throat, cold, cough with sputum, flu-like symptoms, pain, fever, and runny nose $[22,33,34]$.

The four main sources of information about self-medication with antibiotics reported by the participants of this study: previous experience $(23 \%)$, medication prescription (catalog or medicine box) (21\%), consultation with a physician (19.5\%), search On the Internet (10\%), however, a study by health care professionals identified the most important source of antibiotic information [15].A serious finding in the present study was the change in the dose of antibiotics used. Less than half of the subjects changed their dose of antibiotics during the course of self-medication with antibiotics. This is consistent with a similar study [35]. The most important reason for changing the dose of the drug was to accelerate the recovery process. Patients appear to be seeking antibiotics for symptomatic treatment and expect symptoms to improve with decreasing or increasing the dose of the drug. Less than half of the population stopped taking antibiotics after their symptoms disappeared. Only one-third of patients stopped taking antibiotics after taking the drug (which does not necessarily mean ending the course of treatment). More than 10 percent of the population stopped taking antibiotics after completing the treatment period, often learning about a previous doctor's visit and the instructions in the medication brochure about the treatment period.

The results of a study show that people often inform the pharmacist and doctor about the course of antibiotic treatment [35].There are several limitations to this study. The cross-sectional study design limited our preference for causal inference between self-medication with antibiotics and related factors. Although self-reported data may contain a reminder bias, our findings were almost consistent with most other studies. Antibiotic selfmedication may vary from population to population, such as medical students. Given that only one province was surveyed, these results cannot be generalized to the whole country. Selection bias was also established by setting a 6-month limit for antibiotic selfmedication.

\section{Conclusion}

This study showed a very high prevalence of antibiotic selfmedication, which can lead to the development of microbial resistance, antibiotic resistance, and treatment failure. This study also shows that amoxicillin, cephalexin and cefixime were the most commonly used antibiotics for self-medication. Previous experience and lack of time were the most important reasons for selfmedication with antibiotics. Sore throat and cold symptoms (such as cough, runny nose, and fever) were the most important symptoms for self-medication. A relatively large proportion of people changed their antibiotic dose, which was the most important reason for accelerating the recovery of the disease.Educational interventions targeting the general public, pharmacists, and health care students are of particular importance. It was considered a powerful tool to increase the rational use of antibiotics in the community and improve patients' behavior regarding the use of antibiotics. Physicians and pharmacists should be encouraged through national health programs to provide more information about the proper use of antibiotics and their importance to individuals or patients when prescribing or distributing these drugs.

\section{Patents}

This section is not mandatory but may be added if there are patents resulting from the work reported in this manuscript.

Supplementary Materials: The following are available online at www.mdpi.com/xxx/s1, Figure S1: title, Table S1: title, Video S1: title.

Author Contributions: For research articles with several authors, a short paragraph specifying their individual contributions must be provided. The following statements should be used "Conceptualization, HesamAldinVarpaei; methodology, HesamAldinVarpaei; software, HesamAldinVarpaei; validation, HesamAldinVarpaei and Ali Kazemi; formal analysis, HesamAldinVarpaei; investigation, HesamAldinVarpaei and HoseinEsmaeili and Mohammad Yavari and Mohammad mahdiMirememi and Amir Mahdi Farahani and FaezeEsmaeili and Saba Abachi and PariyaOnsory and PedramNouroozi; resources HesamAldinVarpaei and HoseinEsmaeili and Mohammad Yavari and Mohammad mahdiMirememi and Amir Mahdi Farahani and FaezeEsmaeili and Saba Abachi and PariyaOnsory and PedramNouroozi; data curation, Ali Kazemi; writing-original draft preparation, HesamAldinVarpaei.; writing-review and editing, FaezeEsmaeili and Saba Abachi and PariyaOnsory and PedramNouroozi; supervision, Ali Kazemi; project administration, Ali Kazemi.; All authors have read and agreed to the published version of the manuscript.", please turn to the CRediT taxonomy for the term explanation. Authorship must be limited to those who have contributed substantially to the work reported.

\section{Funding}

The study was not financially supported.

\section{Acknowledgment}

The authors are thankful to all the respondents for their voluntary participation in research.

\section{References}

1. Rodrigues Carla F (2020) Self-medication with antibiotics in Maputo, Mozambique: Practices, rationales and relationships. Palgrave Communications 6(1): 1-12.

2. Ayalew Mohammed Biset (2017) Self-medication practice in Ethiopia: A systematic review. Patient preference and adherence 11: 401-413.

3. Eticha Tadele, Kalkidan Mesfin (2014) Self-medication practices in Mekelle, Ethiopia. PloS one 9(5): e97464. 
4. Sisay Mekonnen, Getnet Mengistu, Dumessa Edessa (2018) Epidemiology of self-medication in Ethiopia: A systematic review and meta-analysis of observational studies. BMC Pharmacology and Toxicology 19(1): 56.

5. Fernandes Prabhavathi, Evan Martens (2017) Antibiotics in late clinical development. Biochemical pharmacology 133: 152-163.

6. Piddock Laura JV (2016) Reflecting on the final report of the O'Neil Review on Antimicrobial Resistance. Lancet Infect Dis 16(7): 767-768.

7. Brown Eric D, Gerard D Wright (2016) Antibacterial drug discovery in the resistance era. Nature 529: 336-343.

8. Albawani Sami Mohammed (2017) Self-medication with antibiotics in Sana'a City, Yemen. Tropical Journal of Pharmaceutical Research 16(5): 1195-1199.

9. Alghadeer Sultan, Khalid Aljuaydi, Salmeen Babelghaith, Abdullah Alhammad, Mohammed N Alarifi, et al. (2018) Self-medication with antibiotics in Saudi Arabia. Saudi pharmaceutical journal 26(5): 719 724.

10. Aslam Adeel, Márió Gajdács, Che Suraya Zin, Norny Syafinaz Binti Abd Rahman, Syed Imran Ahmed, et al. (2020) Public awareness and practices towards self-medication with antibiotics among the Malaysian population. A development of questionnaire and pilot-testing. Antibiotics 9(2): 97.

11. Torres NF, B Chibi, LE Middleton, VP Solomon, TP Mashamba Thompsonet, et al. (2019) Evidence of factors influencing self-medication with antibiotics in low and middle-income countries: A systematic scoping review. Public health 168: 92-101.

12. (2015) World Health Organization. Investing to overcome the global impact of neglected tropical diseases: third WHO report on neglected tropical diseases 2015. World Health Organization.

13. Alhomoud Faten, Zainab Aljamea, Reem Almahasnah, Khawlah Alkhalifah, Lama Basalelah, et al. (2017) Self-medication and selfprescription with antibiotics in the Middle East-do they really happen? A systematic review of the prevalence, possible reasons, and outcomes. International Journal of Infectious Diseases 57: 3-12.

14. Jamhour Antoun, Ammar El Kheir, Pascale Salameh, Pierre Abi Hanna, Hanine Mansour, et al. (2017) Antibiotic knowledge and self-medication practices in a developing country: A cross-sectional study. American journal of infection control 45(4): 384-388.

15. Pavydė Eglè, Vincentas Veikutis, Asta Mačiulienė, Vytautas Mačiulis, Kęstutis Petrikonis, et al. (2015) Public knowledge, beliefs and behavior on antibiotic use and self-medication in Lithuania. International journal of environmental research and public health 12(6): 7002-7016.

16. Lv Bing, Zhongliang Zhou, Guiping Xu, Dingkun Yang, Lina Wu, et al. (2014) Knowledge, attitudes and practices concerning self-medication with antibiotics among university students in western China. Tropical Medicine \& International Health 19(7): 769-779.

17. Shah Syed Jawad, Hamna Ahmad, Rija Binte Rehan, Sidra Najeeb, Mirrah Mumtaz, et al. (2014) Self-medication with antibiotics among nonmedical university students of Karachi: A cross-sectional study. BMC Pharmacology and Toxicology 15(1): 74.

18. Al Rasheed Abdulrahman, Umar Yagoub, Hesham Alkhashan, Osama Abdelhay, Ahmad Alawwad, et al. (2016) Prevalence and predictors of self-medication with antibiotics in Al Wazarat health center, Riyadh City, KSA. BioMed research international 2016.

19. Abdulraheem IS, Adeoye Adegboye, AA Fatiregun (2016) Self-medication with antibiotics: empirical evidence from a Nigerian rural population. Journal of Pharmaceutical Research International p. 1-13.
20. Bilal Muhammad, Abdul Haseeb, Mohammad Hassaan Khan, Mohammad Hussham Arshad, Asma Akbar Ladak, et al. (2016) Self-medication with antibiotics among people dwelling in rural areas of Sindh. Journal of clinical and diagnostic research: JCDR 10(5): 0C08-0C13.

21. Owour IJ, Alwar, H Oyugi (2015) Perceptions influencing self-medication with antibiotics and/or antimalarials among the households in Nyalenda B sub-location, Kisumu county, Kenya. Am J Public Health Res 3(3): 116121.

22. Helal RM, HS Abou ElWafa (2017) Self-medication in university students from the city of Mansoura, Egypt. Journal of environmental and public health 2017: 9145193.

23. Al Flaiti Marwa (2014) Evaluation of self-medication practices in acute diseases among university students in Oman. Journal of Acute Disease 3(3): 249-252.

24. Lescure Dominique (2018) Determinants of self-medication with antibiotics in European and Anglo-Saxon countries: A systematic review of the literature. Frontiers in public health $6: 370$.

25. Shaghaghi Abdolreza, Marzieh Asadi, Hamid Allahverdipour (2014) Predictors of self-medication behavior: a systematic review. Iranian journal of public health 43(2): 136-146.

26. Emeka Promise M, Mokhtar Al Omar, Tahir M Khan (2014) Public attitude and justification to purchase antibiotics in the Eastern region Al Ahsa of Saudi Arabia. Saudi Pharmaceutical Journal 22(6): 550-554.

27. Alfadly Saeed, Wajid Ali, Sultan Alghadeer, Salmeen Babelghaith, Abdullah S AMRA, et al. (2017) Self-Medication with Antibiotic amongst Adults Attending Community Pharmacies in Mukalla District, Yemen. Latin American Journal of Pharmacy 36(2): 224-228.

28. Hersh Adam L, Katherine E Fleming-Dutra, Daniel J Shapiro, David Y Hyun, Lauri A Hicks, et al. (2016) Frequency of first-line antibiotic selection among US ambulatory care visits for otitis media, sinusitis, and pharyngitis. JAMA internal medicine 176(12): 1870-1872.

29. Cots Josep M, Juan-Ignacio Alós, Mario Bárcena, Xavier Boleda, José L Cañada, et al. (2015) Recommendations for management of acute pharyngitis in adults. Acta Otorrinolaringologica (English Edn.). 66(3): 159-170.

30. Nepal Gaurav, Shekhar Bhatta (2018) Self-medication with antibiotics in WHO Southeast Asian Region: A systematic review. Cureus 10(4): e2428.

31. Vanitha M, M Vineela, RKP Benjamin (2017) Prescribing pattern of antibiotics in patients attending ENT OPD in a tertiary care hospital. IOSR Journal of Dental and Medical Sciences (IOSR-JDMS) 16(9) : 30-33.

32. Ramachandra K, Narendranath Sanji, HS Somashekar, Abhishek Acharya, Keerthi Sagar J, et al. (2012) Trends in prescribing antimicrobials in an ENT outpatient department of a tertiary care hospital for upper respiratory tract infections. International Journal of Pharmacology and Clinical Sciences 1(1): 15-18.

33. Abdulraheem IS, Adeoye Adegboye, AA Fatiregun (2016) Self-medication with antibiotics: empirical evidence from a Nigerian rural population. Journal of Pharmaceutical Research International pp. 1-13.

34. Kurniawan Kurniawan, Jimmy Posangi, Nancy Rampengan (2017) Association between public knowledge regarding antibiotics and selfmedication with antibiotics in Teling Atas Community Health Center, East Indonesia. Medical Journal of Indonesia 26(1): 62-69.

35. Yousif M, I Abubaker (2015) Prevalence, determinants, and practices of self-medication with antibiotics-a population-based survey in Taif, Kingdom of Saudi Aarabiaksa. Age 228(172): 57-50. 


\section{ISSN: 2574-1241}

DOI: 10.26717/BJSTR.2020.31.005040

Hesam Aldin Varpaei. Biomed J Sci \& Tech Res

\section{(C) (P) This work is licensed under Creative}

Submission Link: https://biomedres.us/submit-manuscript.php

$\begin{array}{ll}\text { BIOMEDICAL } & \text { Assets of Publishing with us } \\ \text { RESEARCHES } & \text { - Global archiving of articles } \\ \text { - Immediate, unrestricted online access }\end{array}$

